\title{
Quantum Transport Modeling of Current Noise in Quantum Devices
}

\author{
TANROKU MIYOSHI, TETSUO MIYAMOTO \\ and MATSUTO OGAWA* \\ Department of Electrical and Electronics Engineering, Kobe University, 1 Rokkodai, \\ Nada, Kobe, 657-8501, Japan
}

\begin{abstract}
We have studied the dependence of noise characteristics on the dimension of electron confinement of quantum devices at low temperature. By using the nonequilibrium Green's function method, we have found that in a double barrier resonant tunneling diode the shot noise is suppressed only around the bias voltage of the resonant tunneling and the noise suppression is more than half of the full shot noise in case of symmetric structures with thin barriers. On the other hand, in the Coulomb staircase characteristics of a quantum dot with equal barriers, the shot noise is suppressed on an average about half of the full shot noise while further drops are observed at the current-step voltages.
\end{abstract}

Keywords: Suppression of shot noise; Pauli principle; Interaction of electrons; Nonequilibrium Green's function; Quantum dot; Resonant tunneling diode

\section{INTRODUCTION}

Shot-noise has attracted a lot of attention in mesoscopic tunneling devices because it gives information on the temporal correlation of the electrons, which is not contained in the timeaveraged transport characteristics such as conductance [1-3]. When the tunneling events are completely random and described by Poisson statistics, the shot noise has its maximum value $2 e I$, where $I$ is the time-averaged current and $e$ is the electron charge. However, when the tunneling event of one electron is correlated to those of other electrons, the shot noise will be suppressed below $2 e I$. There exist two types of correlations of electrons in semiconductor quantum devices. One is the Pauli exclusion principle, which forbids multiple occupancy of the same single particle state. Coulomb interactions are considered another source of correlations among electrons.

In this paper, we have studied the current noise characteristics of quantum devices at low temperature by employing the tight-binding Green's function method based on Keldysh's perturbation theory $[4,5]$. In particular, the dependence of shot noise on the dimension of electron confinement

*Corresponding author. Tel./Fax: +81-78-803-6071, e-mail: miyoshi@eedept.kobe-u.ac.jp 
will be discussed by comparing the noise suppression of the two tunneling devices: a quantum dot and a double barrier resonant tunneling diode (DBRTD). We discussed previously the current noise in semiconductor quantum dots in [6], where the interaction between electrons was assumed to extend over all sites in a dot and it was represented by the retarded self-energy within the HartreeFock approximation. We found that the shot noise at peaks of Coulomb oscillations reduced sharply close to zero when the two barriers were equal, and the Coulomb interaction between the electrons hardly affected the noise in Coulomb oscillations. Furthermore, we demonstrated that the shot noise in Coulomb staircase was also suppressed less than half of the full shot noise, and the noise suppression for the unequal barriers was always smaller than that for equal barriers on an average.

On the other hand, a theory of noise characteristics of a DBRTD system was presented in [1] based on the nonequilibrium Green's function approach, where unfortunately the interaction of electrons was not considered in the calculation, but the computed noise characteristics were qualitatively reasonable. They concluded that the shot noise in a DBRTS was not full, but it was suppressed to a certain degree that depended on the symmetry parameter of the structure. In extremely asymmetric structures, the current is mainly controlled by one barrier and thus the shot noise should be full. On the contrary, it becomes only half of the full shot noise in a symmetric structure. In our paper, to improve the theory [1] the interaction of electrons will be considered within the Hartee approximation by using the quantum mechanical calculation of the charge in an iterative self-consistent solution of the Poisson's equation. It will be shown quantitatively that in a symmetric structure the noise suppression of a DBRTD will not always be limited half of the full shot noise, but will be more than half around the current peak voltage against the prediction of the theory [1].

\section{NON-EQUILIBRIUM GREEN'S FUNCTION APPROACH}

Figure 1 shows the schematic structure of an AlGaAs/GaAs/AlGaAs double barrier resonant tunneling diode used in the tight-binding analysis, which consists of two semi-infinite electrodes with two nondoped spacers, nondoped spacers, nondoped two barriers and a well. In the single-band tight-binding model, the steady state current density $J_{l}$ and the electron density $n_{l}$ at a layer $l$ are represented by using the Fourier transformed correlation function $G^{<}$

$$
\begin{gathered}
J_{l}=\frac{2 e}{\hbar A} \sum_{\boldsymbol{k}_{\|}} \int \frac{d E}{2 \pi} 2 \operatorname{Re}\left\{t_{l, l+1} G_{l+1, l}^{<}\left(\boldsymbol{k}_{\|}, E\right)\right\} \\
n_{l}=-\frac{2 i}{A \Delta} \sum_{\boldsymbol{k}_{\|}} \int \frac{d E}{2 \pi}\left\{G_{l, l}^{<}\left(\boldsymbol{k}_{\|}, E\right)\right\}
\end{gathered}
$$

where $\boldsymbol{k}_{\|}$is a transverse wave vector, and $t_{l, m}$ denotes the hopping energy between the $l$ th and $m$ th layers. $A$ and $\Delta$ are the cross section of the device and the layer spacing, respectively.

The power spectrum of noise current obtained by the Fourier transform of the current correlation

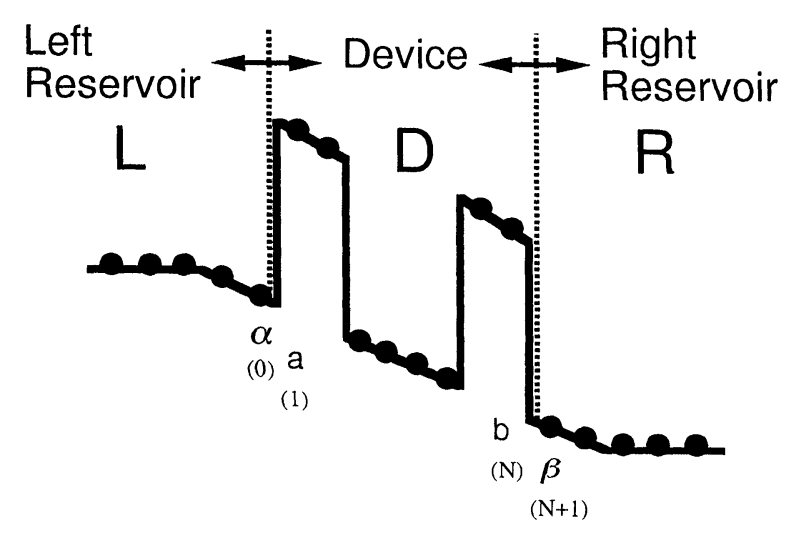

FIGURE 1 Schematic structure of a AlGaAs/GaAs/AlGaAs double barrier resonant tunneling diode (DBRTD) used in the tight-binding Green's function analysis. 
function is given at low frequency as

$$
\begin{aligned}
S_{l}(0)=\frac{4 e^{2} t_{l, l+1}^{2}}{\hbar A} \sum_{\boldsymbol{k}_{\|}} \int_{-\infty}^{\infty} \frac{d E}{2 \pi} \\
{\left[G_{l, l}^{<}\left(E, \boldsymbol{k}_{\|}\right) G_{l+1, l+1}^{>}\left(E, \boldsymbol{k}_{\|}\right)\right.} \\
\quad+G_{l+1, l+1}^{<}\left(E, \boldsymbol{k}_{\|}\right) G_{l, l}^{>}\left(E, \boldsymbol{k}_{\|}\right) \\
\quad-G_{l, l+1}^{<}\left(E, \boldsymbol{k}_{\|}\right) G_{l, l+1}^{>}\left(E, \boldsymbol{k}_{\|}\right) \\
\left.-G_{l+1, l}^{<}\left(E, \boldsymbol{k}_{\|}\right) G_{l+1, l}^{>}\left(E, \boldsymbol{k}_{\|}\right)\right]
\end{aligned}
$$

In the numerical calculation, the quantum mechanical electron density given by Eq. (2) is used in the iterative self-consistent solution of Poisson's equation to consider the interaction of electrons within the Hartree approximation.

On the other hand, the analysis of the current fluctuation of a quantum dot is much the same as mentioned above, but there are two major different points. One is the perfect quantization of electron energy in a quantum dot, which removes the calculation of the sum in terms of $\boldsymbol{k}_{\|}$in Eqs. (1)-(3). Second, since the interaction of electrons inside the dot plays a crucial role in the transport, the intradot interaction is well incorporated in terms of self-energy within the Hartree-Fock approximation. In addition, to model the quantum transport in the barrier more precisely than that implemented in [5], we proposed to consider the fact that the transfer coefficient through the barrier depends on the energy and further, an electron inside the barrier can make excursions into the lead [6].

\section{NOISE CHARACTERISTICS OF QUANTUM DEVICES}

\section{Double Barrier Resonant Tunneling Diode}

Figures 2(a)-(c) show the calculated $I-V$ characteristics, the shot noise power, and the noise power ratio, respectively, for a $\mathrm{Al}_{0.4} \mathrm{Ga}_{0.6} \mathrm{As} /$ $\mathrm{GaAs} / \mathrm{Al}_{0.4} \mathrm{Ga}_{0.6} \mathrm{As}$ DBRTD with symmetric structure. Here, the noise power ratio is defined
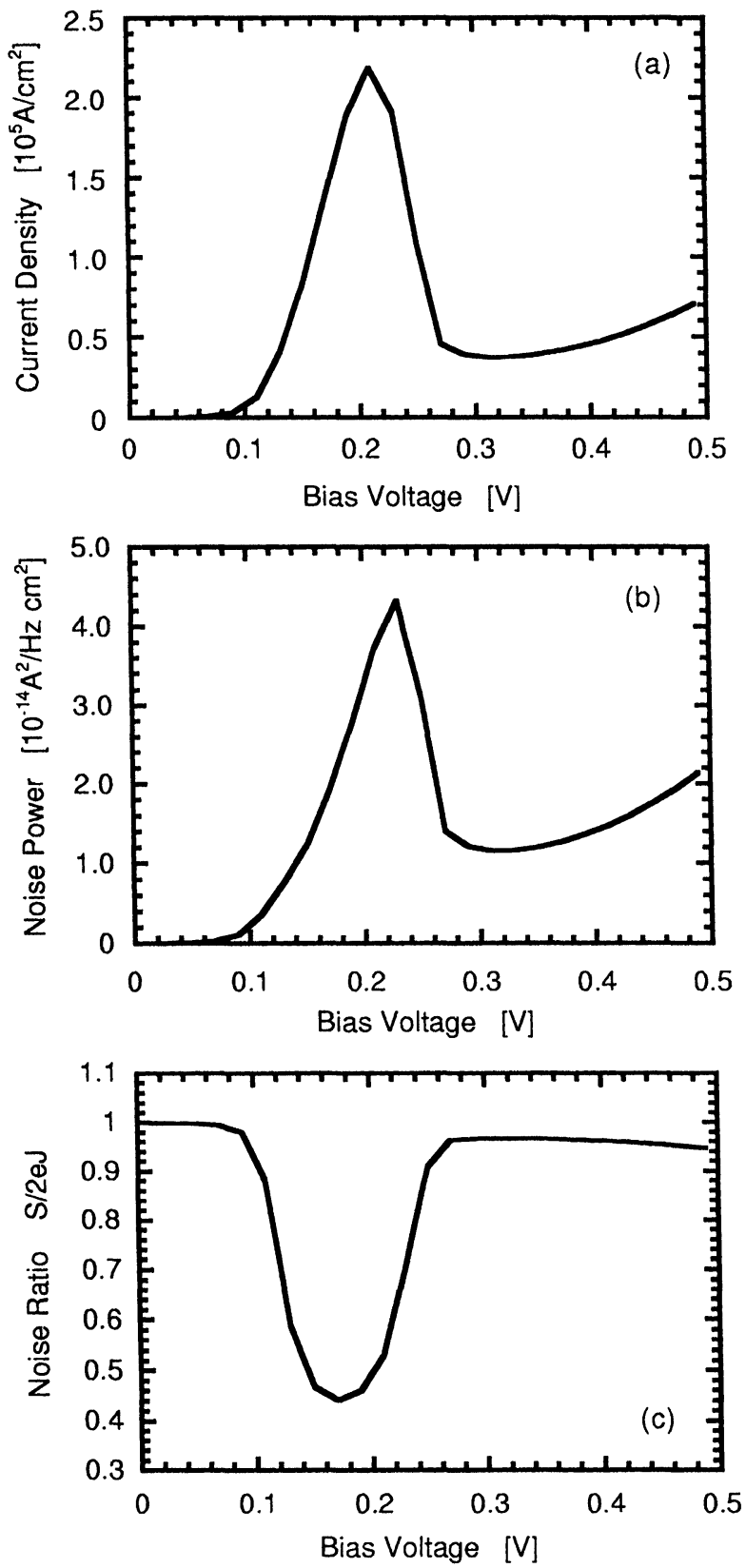

FIGURE $2 I-V$ characteristics of a double barrier resonant tunneling diode with symmetric structure (a), the shot noise power (b), and the noise power ratio defined by the noise power divided by full shot noise (c).

by the noise power divided by full shot noise. The thickness of barrier and that of well are 8 layers and 20 layers, respectively. In the calculation, the 

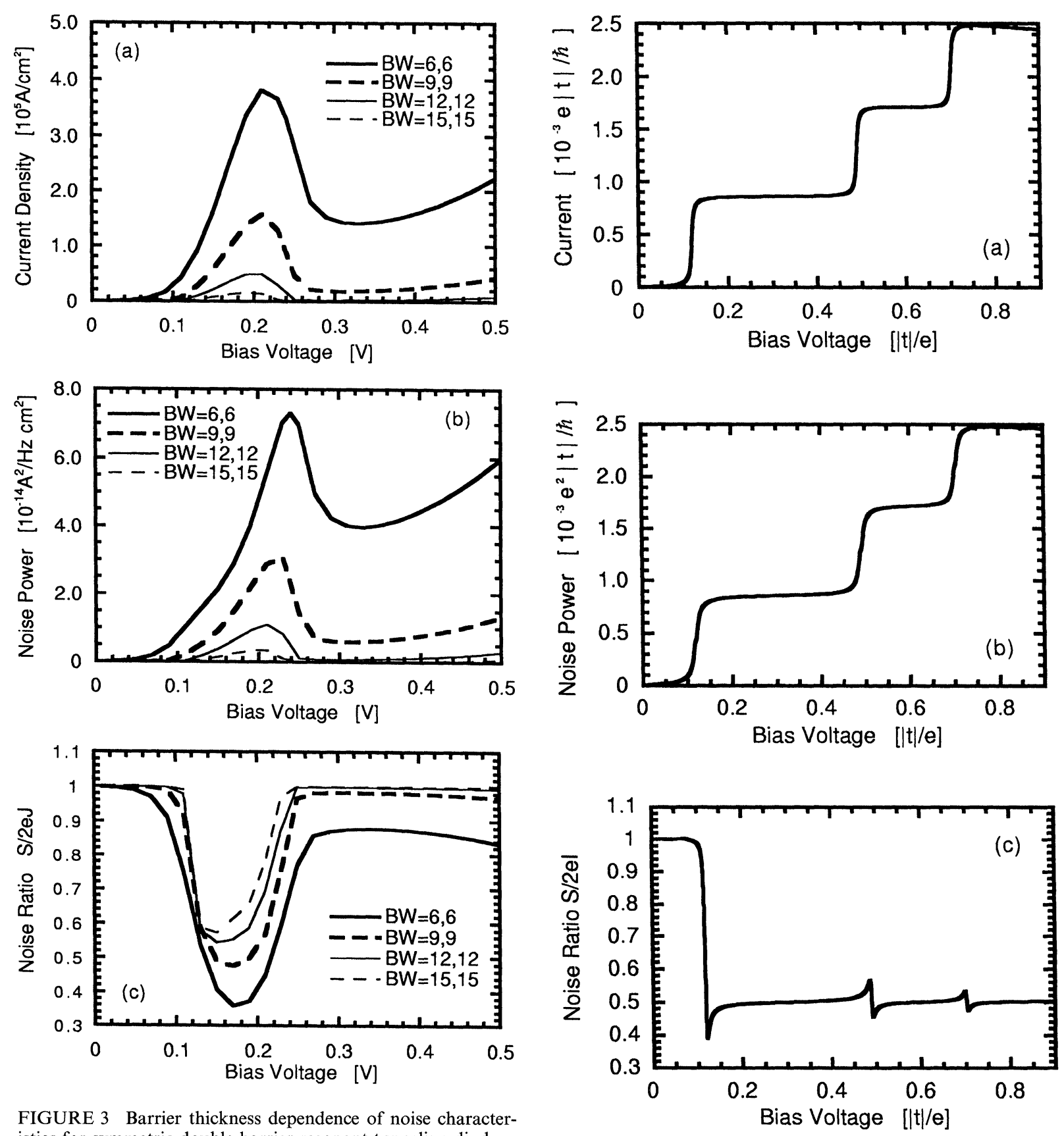

FIGURE 3 Barrier thickness dependence of noise character-
istics for symmetric double barrier resonant tunneling diodes.

doping density in the electrodes is assumed $1.0 \times$ $10^{18}\left[\mathrm{~cm}^{-3}\right]$, and the temperature is $4.2 \mathrm{~K}$. The suppression of shot noise is observed only around the bias voltage of the resonant tunneling. The noise suppression is found to be maximum at a

FIGURE 4 Coulomb staircase characteristics of a quantum dot with equal barriers (a), the shot noise power (b), and the noise power ratio $(c)$.

bias voltage close to the current peak voltage. Furthermore, in the study of the barrier thickness dependence of noise characteristics in symmetric 
structure, we have found that the noise suppression is enhanced as the thickness of barrier decreases as shown in Figure 3, where the barrier thickness changes from 6 layers to 15 layers. More interestingly, the noise suppression of a DBRTD is not always limited to half of the full shot noise, but reaches more than half against the prediction of the simple theory [1].

\section{Quantum Dot}

In Figure 4, the Coulomb staircase characteristics, the shot noise power, and the noise power ratio for a dot with equal barriers are shown as functions of bias voltage, where $t$ used as the unit of energy denotes the hopping energy. The averaged $I-V$ characteristics have step like increases of current with voltage. At each subsequent step in current, the number of current-carrying electrons increases by one. The tunneling current is always flowing above the bias voltage where the current starts to flow after overcoming the initial Coulomb blockade. In this bias region, since the tunneling of one electron is correlated to those of other electrons due to the Pauli exclusion principle, the shot noise is suppressed on an average about half of the full shot noise while further drops in noise ratio are observed at the current step voltages as shown in Figure 4(c).

\section{CONCLUSION}

We have studied the current noise characteristics of quantum devices at low temperature, particularly, the dependence of noise suppression on the dimension of electron confinement by comparing the two tunneling devices: a quantum dot and a double barrier resonant tunneling diode (DBRTD). By using the nonequilibrium Green's function method, we have found that the suppression of shot noise is observed in DBRTD only around the bias voltage of the resonant tunneling. The noise suppression is found to be maximum at a bias voltage a little lower than the current peak voltage. Furthermore, we have found that the noise is suppressed more than half of the full shot noise in case of the symmetric DBRTD with thin barriers. On the other hand, in the Coulomb staircase characteristics of a quantum dot with equal barriers, the shot noise is always suppressed on an average about half of the full shot noise while further drops in noise ratio are observed at the current step voltages.

\section{References}

[1] Chen, L. Y. and Ting, C. S. (1991). Theoretical investigation of noise characteristics of double-barrier resonanttunneling systems, Phys. Rev., B 43(5), 4534-4537.

[2] Birk, H., de Jong, M. J. M. and Schonenberger, C. (1995). Shot noise suppression in the single-electron tunneling regime, Phys. Rev. Lett., 75(8), 1610-1613.

[3] Hershfield, S., Davies, J. H., Hyldgaard, P., Stanton, C. J. and Wilkins, J. W. (1993). Zero frequency current noise for the double-tunnel-junction Coulomb blockade, Phys. Rev., B 47, 1967-1979.

[4] Lake, R., Klimeck, G., Brown, R. C. and Jovanovic, D. (1997). Single and multiband modeling of quantum electron transport through layered semiconductor devices, J. Appl. Phys., 81(12), 7845-7869.

[5] Henrickson, L. E., Glick, A. J., Bryant, G. W. and Barbe, D. F. (1994). Nonequilibrium-Green's function theory of transport in interacting quantum dots, Phys. Rev., B 50(7), $4482-4496$.

[6] Wang, Z., Iwanaga, M. and Miyoshi, T. (1998). Current noise in semiconductor quantum dots, Jpn. J. Appl. Phys., 37(11), 5894-5901. 

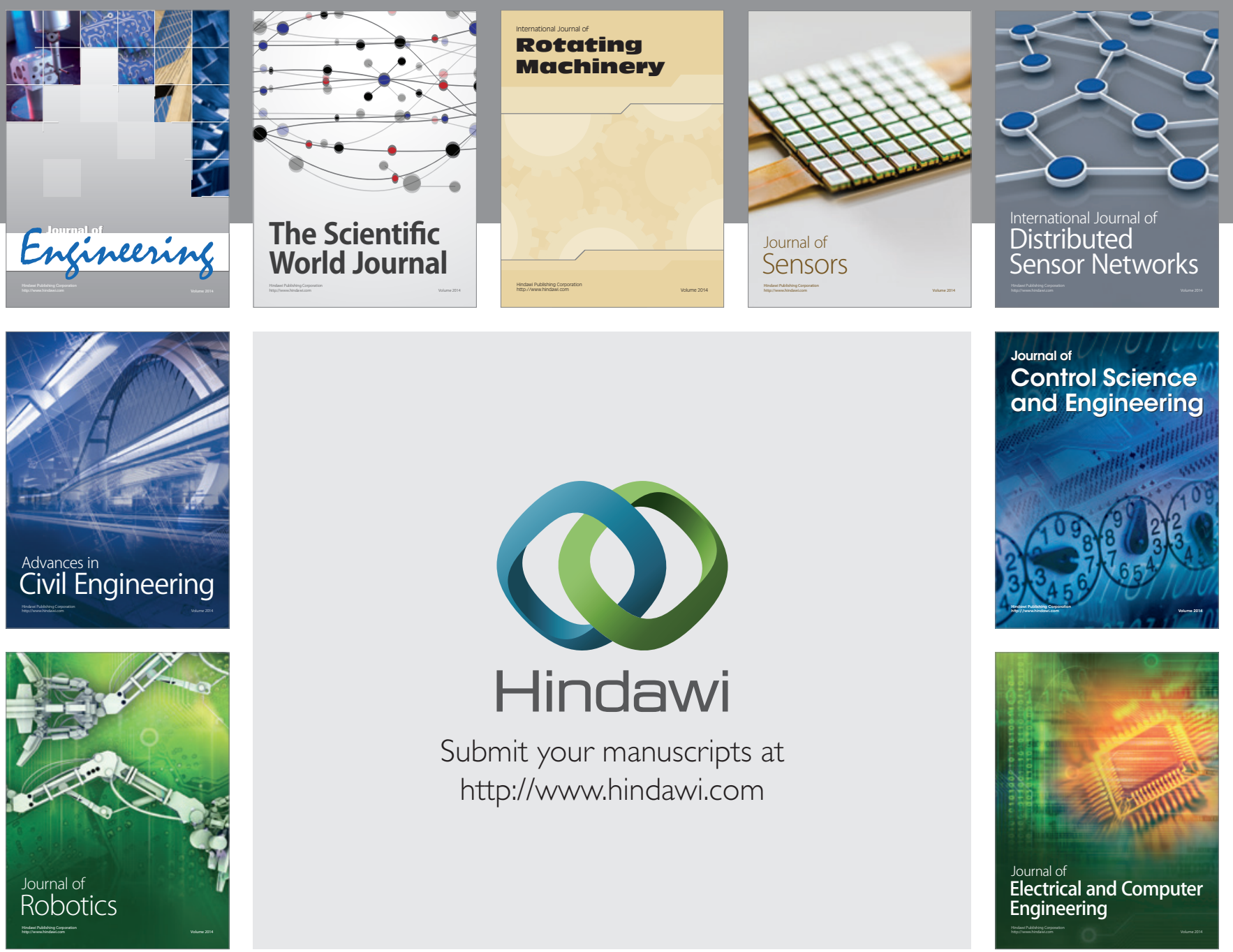

Submit your manuscripts at

http://www.hindawi.com
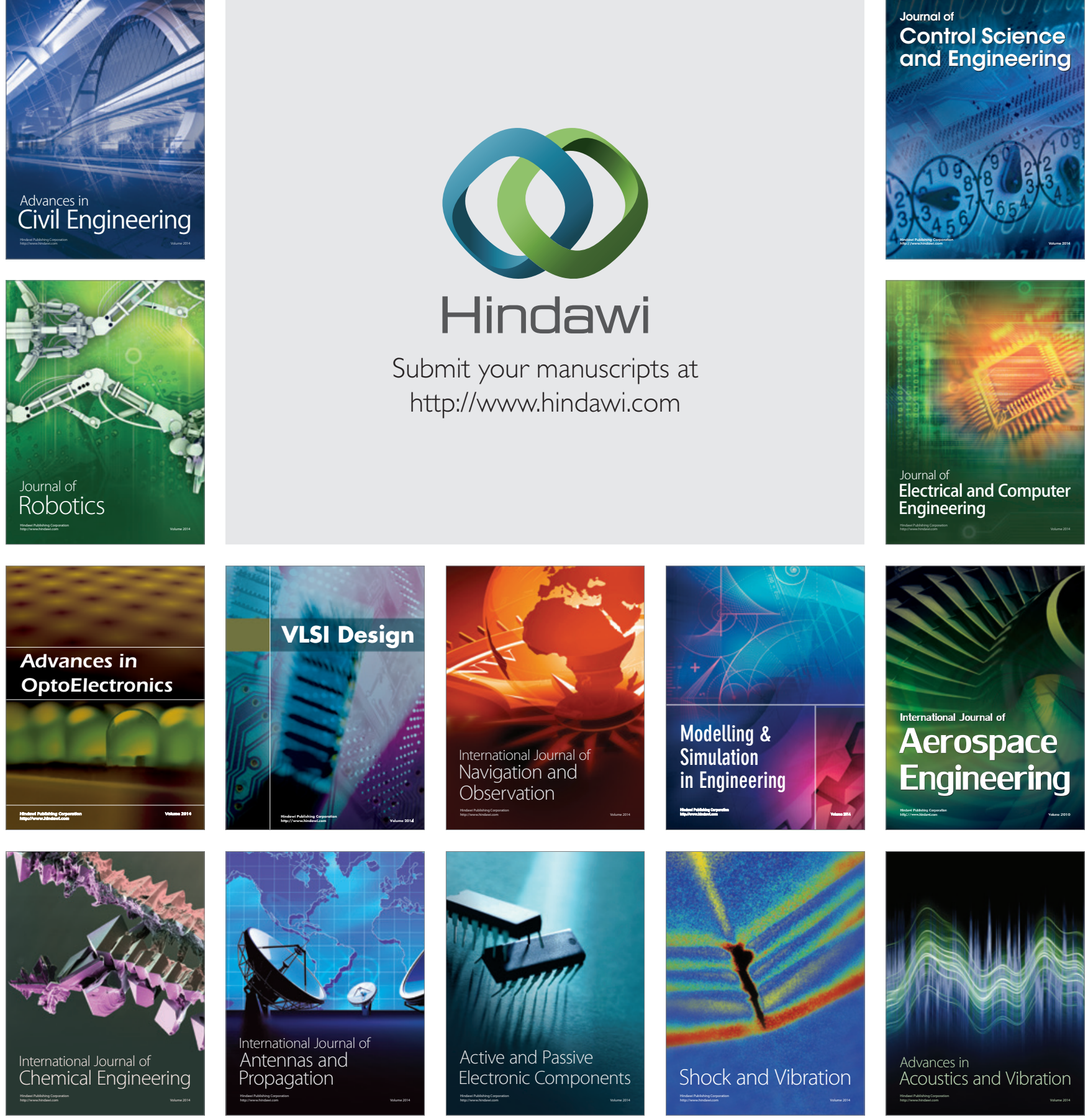